\title{
The Nonlinear Least Square Fitting for Rotation Curve of Orion Dwarf Spiral
}

(Penyuaian Kuasa Dua Terkecil Tak Linear bagi Putaran Lengkung Orion Kerdil Berpilin)

\author{
N. HASHIM*,Z.Z. ABIDIN, U.F.S.U. IBRAHIM, M.S.R. HASSAN, Z.S. HAMIDI, R. UMAR \& Z.A. IBRAHIM
}

\begin{abstract}
The basis of the nonlinear least square fitting is to fit the nonlinear rotation curve model with the observed rotation curve of the Orion dwarf galaxy. It has been the most powerful tool to study the distribution of dark matter in galaxies where it is used to obtain the proper mass model of a galaxy. In this paper, we present the rotation curve fit of Orion dwarf galaxy, corrected for asymmetric drift by using the gradient method of nonlinear least square. Our results showed an excellent agreement between the mass models of cored halo profile with the observed rotation curve. Thus, we can estimate the value of disk mass, MD; the core radius, $r_{0}$ and core density, $r_{o}$ of the galaxy with 1 -s of uncertainty. We finally indicated the dark matter halo distribution as cored dark matter halo with density, $3.9 \times 10^{6} \mathrm{M}_{\ddot{Y}} \mathrm{kpc}^{-3}$.
\end{abstract}

Keywords: Dwarf spiral - rotation curve - dark matter; fitting; galaxies; gradient method

ABSTRAK

Asas kepada penyuaian kuasa dua terkecil tak linear adalah menyuaikan model putaran lengkung dengan cerapan putaran lengkung galaksi Orion kerdil. Ia telah menjadi kaedah yang paling berkuasa dalam kajian taburan jirim gelap di dalam galaksi dan ia digunakan untuk mendapatkan model jisim yang bersesuaian untuk suatu galaksi. Di dalam artikel ini, kami mempersembahkan suaian putaran lengkung bagi galaksi Orion kerdil yang telah dibetulkan pecutan asimetri dengan menggunakan kaedah kecerunan daripada kuasa dua terkecil tak linear. Keputusan yang kami peroleh menunjukkan keselarian yang cemerlang antara model jisim profil halo teras dengan cerapan putaran lengkung. Justeru, kami boleh menganggarkan nilai jisim cakera, MD; jejari teras, $r_{0}$ dan ketumpatan teras, $r_{o}$ bagi suatu galaksi di bawah 1-s ketidakpastian. Kami juga akhirnya menunjukkan bahawa taburan halo jirim gelap adalah halo jirim gelap berteras dengan ketumpatan, $3.9 \times 10^{6} \mathrm{M}_{\ddot{Y}} \mathrm{kpc}^{-3}$.

Kata kunci: Galaksi; kaedah kecerunan; kerdil berpilin - putaran lengkung - jirim gelap; penyuaian

\section{INTRODUCTION}

It is widely accepted that the Orion dwarf galaxy is a barred spiral type $d$ or can also be written as $S B d$. It is observed at 5.4 Mpc (adopted distance by Vaduvescu et al. 2005) from the observer and found to be an excellent laboratory to apply the rotation curve fit (Cannon et al. 2010). The data was obtained from the KPNO 0.9-m optical telescope and VLAHI spectral line observations. The $21 \mathrm{~cm}$ HI surface brightness and kinematics have recently been published by Cannon et al. (2010). Work by Frusciante et al. (2012) shows that the rotation curve with cored dark matter halo is fitted well on the Orion dwarf galaxy. However in this paper, we attempt to reproduce the observed rotation curve of Orion with the same profile of dark matter halo as Frusciante et al. (2012) but with a different disc scale length, adopted from Vaduvescu et al. (2005). The was derived from the fitting of surface brightness profile of Orion in the near-infrared (NIR) sources. We employ the value of $\mathrm{kpc}$ at the adopted distance $5.4 \mathrm{Mpc}$. While the disk scale length used in the paper Frusciante et al. (2012), is kpc. Our new disc scale length, . The length scale is indirectly indicates the luminosity of a galaxy. Therefore, we have considered the galaxy is less luminous (Frusciante et al. 2012). It is strongly supported by the fact that Orion dwarf spiral is fainter galaxy (Cannon et al. 2010). Thus, we present the recent best-fit rotation curve of Orion dwarf galaxy with using gradient method of nonlinear least square fitting.

The observed rotation curve of Orion dwarf galaxy can be seen in Figure 1. It demonstrates several important properties, namely the solid-body rotation up to $3.7 \mathrm{kpc}$, then starting to flatten and slowly rising until the detection limit in the outer disk, $6.8 \mathrm{kpc}$. The slowly rising rotation curve represents the dark matter dominated at all radii (Carignan \& Beaulieu 1989; Côté et al. 2000). It can be shown by rotation curve analysis (Longair 2008). This inferred evidence is crucial to explain the existence of dark matter in the Orion dwarf galaxy.

\section{NONLINEAR LEAST SQUARE FITTING}

Fitting the nonlinear functions to data is sometimes more towards an art of minimizing the function of rather than a science itself. However, this analysis could possibly give clues on the value of parameters in the rotation curve fitting. Generally, the first step of a fitting is to find the right 


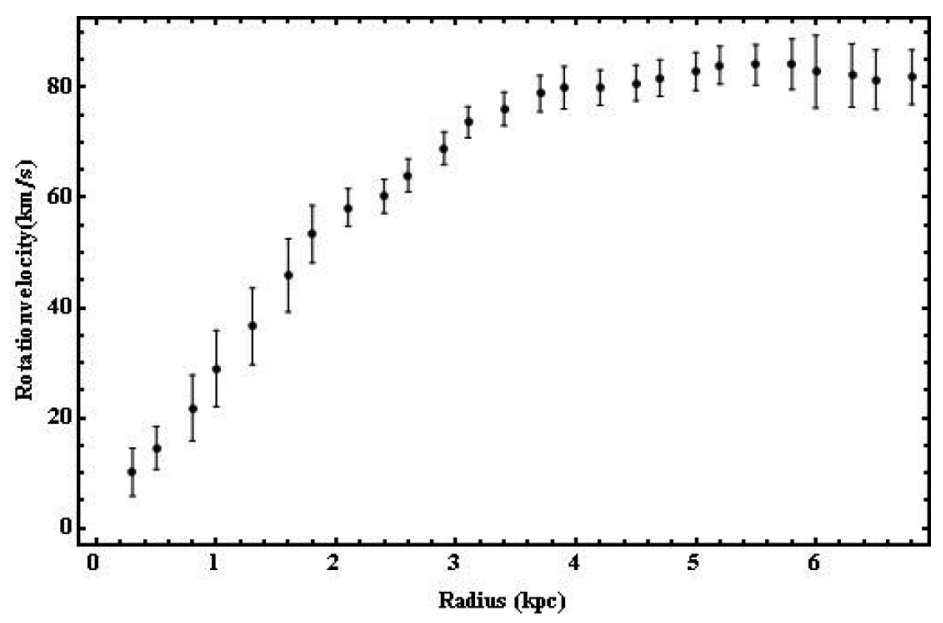

FIGURE 1 . The rotation curve of Orion dwarf galaxy with error bars of uncertainty

starting values for the parameters. Then we plot the data with curves that are computed from several trial values of the parameters. The appropriate values of the parameters will approximately minimize and indicate the best-fit. In particular, the large value of indicates a poor-fit and the model has not fully captured the observed data. For a three parameter fit, the parameters obtained by this procedure are sufficiently precise and no further searching is required. In this section we will fit our nonlinear fitting model that involves three free parameters with the observed rotation curve of Orion dwarf galaxy. Our nonlinear fitting model is a combination of a rotation velocity from the Freeman stellar disk by Freeman (1970) and Burkert dark matter halo by Burkert (1995) as follows:

$$
\begin{aligned}
V_{\text {model } 1}= & \left\{\frac{5.1 G M_{D} x^{2}}{R_{D}}\left(I_{0} K_{0}-I_{1} K_{1}\right)+\frac{6.4 G \rho_{0} r_{0}^{3}}{r}\right. \\
& \left.\left(\ln \left[(1+a)\left(1+a^{2}\right)^{0.5}\right]-\tan ^{-1} a\right)\right\}^{0.5},
\end{aligned}
$$

where $G$ is the universal gravitational constant, $I_{n}$ and $K_{n}$ are the modified Bessel functions of the first and second kinds computed at $1.6 x$ and $x=\frac{r}{3.2 R_{D}} . R_{D}$ is the disk scale length with value, kpc. $a$ is defined as $a=\frac{r}{r_{0}}$ where $r$ is the radius from the centre of the gravitational potential. The three free parameters of the fitting, $M_{D}, \rho_{o}, r_{o}$, and $r_{o}$ are the disk mass, core density, and core radius, respectively.

In this paper, we also need to test another similar nonlinear fitting model where we used the same stellar disk rotation velocity from the first model but with different halo profile, that is NFW dark matter halo by Navarro et al. (1997). It is tested to justify whether cuspy halo will be the right term to explain the distribution of dark matter halo profile. The corresponding model is represented as follows:

$$
V_{\text {halo }}^{2}=\frac{4 \pi G \rho_{0} r_{s}^{3}}{r}\left\{\ln \left(1+\frac{r}{r_{s}}\right)-\frac{r / r_{s}}{r / r_{s}+1}\right\} \text {. }
$$

This dark matter halo model illustrates a different halo structure than Burkert halo profile, where the halo is approximately isothermal over a large range of radii, but a bit shallower than $r^{-2}$ near the centre and steeper than $r^{-2}$ near the virial radius, $r_{s}$ (Navarro et al. 1997). Now we have a second model to be considered in our fitting, that is:

$$
\begin{aligned}
V_{\text {model } 2}= & \left\{\frac{5.1 G M_{D} x^{2}}{R_{D}}\left(I_{0} K_{0}-I_{1} K_{1}\right)+\right. \\
& \left.\frac{12.6 G \rho_{0} \rho_{s}^{3}}{r}\left[\ln \left(1+a^{\prime}\right)-\frac{a^{\prime}}{a^{\prime}+1}\right]\right\}^{0.5},
\end{aligned}
$$

where $a^{\prime}=\frac{r}{r_{s}}$. Both rotation curve models in (1) and (3) are necessary to be modified by adding another component, namely the rotational velocity of neutral hydrogen (HI) since it is the main component that contributes to the mass of the galaxy. We also consider the asymmetric drift to the observed rotation curve; see column (3) in Table 1 . In principle, the rotational velocity of HI needs to be corrected for the pressure gradients in the gas to derive the true rotation velocity (Weldrake et al. 2003). The asymmetric drift correction is governed by the Jeans equations in Binney and Tremaine (2011), gives, $V_{c}^{2}=V_{H I}^{2}-\sigma^{2}\left(\frac{\partial \ln \rho}{\partial \ln r}+\frac{\partial \ln \sigma^{2}}{\partial \ln r}\right)$, where $V_{c}$ is the corrected circular velocity, $\mathrm{V}_{H I}^{2}$ is the observed gas rotation velocity, $\sigma$ the velocity dispersion in the gas, and $\rho$ the volume density (Gentile et al. 2007). Finally, we have the new data of the observed rotation curve, $\mathrm{V}_{\text {NewData }}^{2}$ in column (4). We fit each model in (1) and (3) to the observed rotation curve, corrected for asymmetric drift. In fitting the model, it has become customary to test the goodness-of-fit to justify which model will be the best model for our observational data. We do the test by minimizing the weighted sum of squares of the deviation between the observed data and the model fit. The goodness-of-fit parameter, $\chi^{2}$ to be minimized is Bevington and Robinson (1969):

$$
\chi^{2} \equiv \sum_{i=1}^{N}\left\{\frac{1}{\sigma_{i}^{2}}\left[y_{i}-y\left(x_{i}, a, b, c\right)\right]^{2}\right\}
$$


where there are $N$ observed data points $\left(x_{i} ; y_{i}\right), x_{i}$ is the radius from the galactic centre, $y_{i}$ is the observed rotation curve, $\sigma_{i}$ is the uncertainty in $y_{i}, y\left(x_{i} ; a ; b ; c\right)$ are the values of the model function calculated at $x_{i}$, and $a ; b ; c$; are the fit parameters. For nonlinear fitting problems, there are several ways of finding minimum for the function $\chi^{2}$ given in (4). Thus, we construct routines of nonlinear fitting for gradient method. There may be more than one minimum of the $\chi^{2}$ function within a reasonable range values for the parameters. In our case, we can set of the value of the parameters are non-negative since it may converge toward solutions that are physically unreasonable. Furthermore, all parameters have their own typical value and we can justify the starting values for each parameter. In gradient method, all the parameters $a ; b ; c$ are incremented simultaneously with relative magnitudes adjusted so that the resultant direction of travel in parameter space is along the gradient of $\chi^{2}, \nabla \chi^{2}=\sum_{j=1}^{n}\left[\frac{\partial \chi^{2}}{\partial a_{j}} \hat{a}_{j}\right]$ where $\hat{a}_{j}$ indicates a unit vector in the direction of the $a$ coordinate axis.

Computation of $\chi^{2}$ is crucial for our fitting to determine if a fit is good. Yet, the true expectation value for $\chi^{2}$ is

$$
\left\langle\chi^{2}\right\rangle=v=N-N_{c}
$$

where $v$ is the number $N$ of data points minus the number and $N_{c}$ of fit parameters in (4). It is also known as the number of degrees of freedom. To derive the best expected value of $\chi^{2}$, the various combinations of the parameters were fitted in (1) and (3). In this case, we would expect to get $\chi^{2}=23$. Nevertheless, it is a fact that in any physical observation, it is impossible to get an ideal agreement between the observed data and our expectation that leads to $\chi^{2}=0$. Another famous indicator for the goodness-of-fit test is to use the reduced chi-square, $\chi_{\text {red }}^{2}=\frac{\chi^{2}}{v}$ with expectation value of $\left\langle\chi_{\text {red }}^{2}\right\rangle=1$.

\section{RESULTS AND DISCUSSION}

In this section, we may look at the results obtained when we do the fitting of the observation data with the rotation curve model in (1) and (3), HI data, and corrected asymmetric drift in Table 1. The curve fitting results are illustrated in Figures 2 and 3, while the values of the free parameters: disk mass, $M_{D}$; core density, $\rho_{o}$; core radius, $r_{o}$; and scale radius, $r$ together with the $1-\sigma$ standard errors are presented in Table 2. The following numerical results of statistical analysis are found: back to the goodness-of-fit in (4), (i) the first model in (1) gives the value of $\chi^{2} \approx 23$, which meets the expected value of $\chi_{\text {red }}^{2} \approx 1$. (ii) The second model inequation (3) gives $\chi^{2} \approx 55$ which is far from the value $v$, the number of degrees of freedom. Thus, we obtained $\chi_{\text {red }}^{2}$ $>1$, i.e. $\chi_{\text {red }}^{2}=2.4$ (Table 2 ). This value of $\chi_{\text {red }}^{2}$ indicates whether it is a poor observation or incorrect choice of modelling or incorrect assignment of uncertainties. Notice also that the rotation curve fit results in Figure 3 shows a great discrepancy between the observational data with the $V_{\text {model2 }}$ starting from $r=5.8 \mathrm{kpc}$. Hence, $V_{\text {model } 1}$ agrees with
TABLE 1 . HI velocity, asymmetric drift velocity and the new data of the observed rotation curve

\begin{tabular}{|c|c|c|c|}
\hline $\begin{array}{c}\text { Radius (kpc) } \\
\text { (1) }\end{array}$ & $\begin{array}{c}\left(\mathrm{km} \mathrm{s}^{-1}\right) \\
(2)\end{array}$ & $\begin{array}{c}\left(\mathrm{km} \mathrm{s}^{-1}\right) \\
(3)\end{array}$ & $\begin{array}{c}\left(\mathrm{km} \mathrm{s}^{-1}\right) \\
(4)\end{array}$ \\
\hline 0.3 & -2.5 & -12.2 & 10.2 \\
\hline 0.5 & -4.9 & -17.3 & 14.6 \\
\hline 0.8 & -6.7 & -17.4 & 21.8 \\
\hline 1.1 & -6.8 & -13.5 & 28.9 \\
\hline 1.3 & -3.9 & -6.0 & 36.6 \\
\hline 1.6 & 3.1 & 4.9 & 45.9 \\
\hline 1.8 & 15.5 & 19.5 & 53.3 \\
\hline 2.1 & 33.8 & 37.4 & 58.2 \\
\hline 2.4 & 59.0 & 58.2 & 60.2 \\
\hline 2.6 & 87.8 & 81.1 & 64.0 \\
\hline 2.9 & 118.6 & 104.9 & 69.0 \\
\hline 3.1 & 170.0 & 128.0 & 73.7 \\
\hline 3.4 & 220.0 & 149.0 & 76.1 \\
\hline 3.7 & 240.0 & 166.8 & 78.9 \\
\hline 3.9 & 260.0 & 180.5 & 79.9 \\
\hline 4.2 & 270.0 & 189.9 & 79.9 \\
\hline 4.5 & 275.0 & 194.9 & 80.8 \\
\hline 4.7 & 280.0 & 195.9 & 81.7 \\
\hline 5.0 & 280.0 & 193.1 & 82.9 \\
\hline 5.2 & 250.0 & 187.1 & 84.0 \\
\hline 5.5 & 237.0 & 178.1 & 84.1 \\
\hline 5.8 & 226.0 & 166.6 & 84.2 \\
\hline 6.0 & 220.0 & 153.0 & 82.9 \\
\hline 6.3 & 214.7 & 137.8 & 82.1 \\
\hline 6.5 & 208.5 & 121.5 & 81.4 \\
\hline 6.8 & 201.9 & 104.9 & 81.8 \\
\hline
\end{tabular}

the observed data of Orion dwarf galaxy with asymmetric drift correction.

Furthermore, we also plot the residuals to visually inspect the goodness of our fitting. The patterns of the residuals should be checked for a sign of bad fit. Figure 4(a) shows a significant scatter of the residuals around zero where the range of $3.4 \leq V_{\text {res }} \leq-10.7$ of the fitting for $V_{\text {model } 1}$ in (1). While Figure 4(b) shows a wider scatter of residuals around the range of $6.86 \leq V_{\text {res }} \leq-14.8$ of the fitting for $V_{\text {model }}$ in (3) which gives a sign of poor-fit. The well-fitted $V_{\text {model }}$ can also be supported by looking at the predicted curve fall in the confidence bands. The confidence bands are calculated from the confidence level and the standard error of the fit. The confidence bands for both models can be found in Figures 5 and 6 . It gives us a visual sense of how well our data define the best-fit curve. In this case, the purple solid lines represent $68 \%$ confidence level and the purple shaded area is the $68 \%$ confidence bands for the expected value of rotation velocity associated with the radius from the galactic centre. The confidence bands can also be interpreted as there is about $95 \%$ certainty that the observed rotation curves with error-bars lie within the blue bands. The same interpretation goes to $99 \%$ confidence bands in the green shaded area. Overall, we can see that only Figure 6 


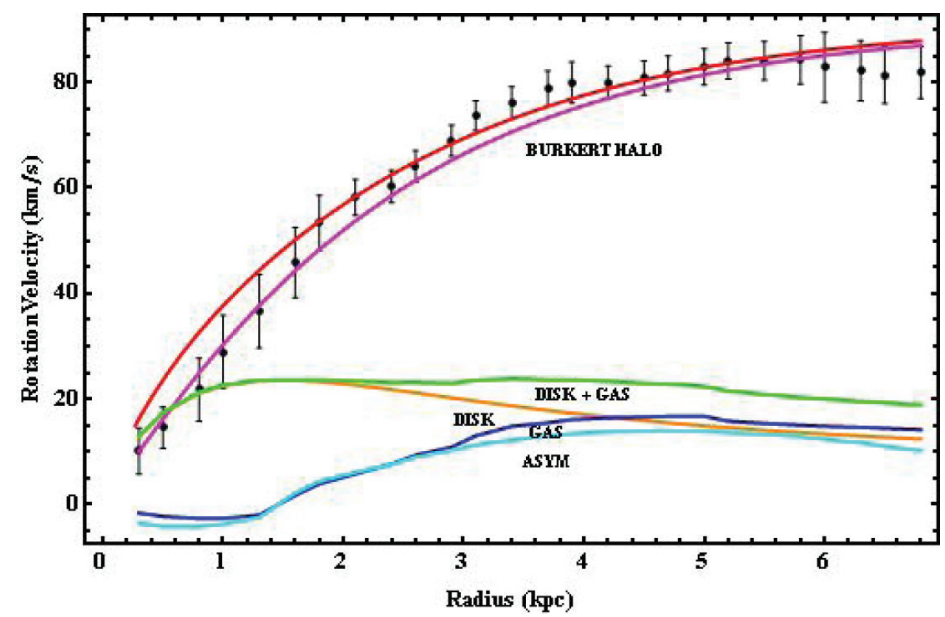

FIGURE 2. The rotation curve decomposition for Orion dwarf galaxy: Asymmetric drift correction (cyan curve), rotation velocity from gas contribution (dark blue curve), rotation velocity from the stellar disk contribution (orange curve), stellar disk and gas rotation velocity (green curve), Burkert halo contribution (pink curve) and the modelled (red curve) of the observed rotation curve (error bar plot)

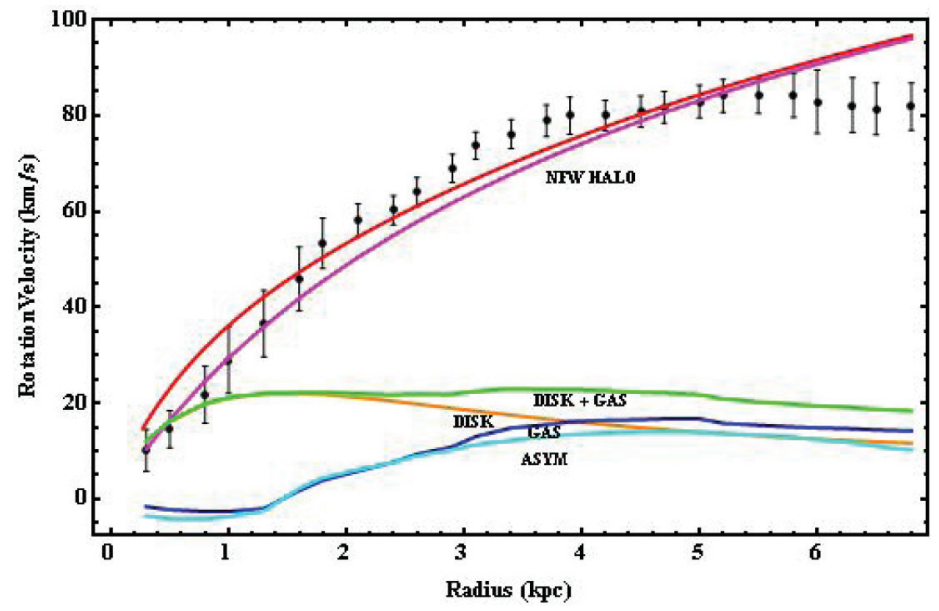

FIGURE 3. The rotation curve decomposition for Orion dwarf galaxy: Asymmetric drift correction (cyan curve), rotation velocity from gas contribution (dark blue curve), rotation velocity from the stellar disk contribution (orange curve), stellar disk and gas rotation velocity (green curve), NFW halo contribution (pink curve), and the modelled (red curve) of the observed rotation curve (error bar plot)

TABLE 2. The standard error for the model's parameters with $\chi_{\text {red }}^{2}$ of the fitting

\begin{tabular}{lccccc}
\hline Model & $M_{D}\left(M_{\odot}\right)$ & $\rho_{0}\left(M_{\odot} \mathrm{kpc}^{-3}\right)$ & $r_{0}(\mathrm{kpc})$ & $r_{s}(\mathrm{kpc})$ & $\chi_{\text {red }}^{2}$ \\
\hline Burkert & $(2.3 \pm 1.1) \times 10^{8}$ & $(6.5 \pm 1.1) \times 10^{7}$ & $3.2 \pm 0.4$ & - & $\approx 1$ \\
NFW & $(2.0 \pm 1.9) \times 10^{8}$ & $(2.4 \pm 2.4) \times 10^{7}$ & - & $2.2 \pm 1.1$ & 2.4 \\
\hline
\end{tabular}

shows one data point with error-bar that is outside those confidence bands, that is it is at kpc. Concerning the dark matter distribution, we found a consistency between the observed rotation curve with the model having the Burkert dark matter halo profile (1), which shows that the dark halo has a central constant-density core, $\rho b=3.9 \times 10^{6} \mathrm{M}$ $\odot \mathrm{kpc}^{-3}$. This result provides important information on the origin and nature of dark matter in the dwarf galaxies. In particular, we have sought the solution of 'core-cusp problem' for density profile of dark matter in the Orion dwarf galaxy.

\section{CONCLUSION}

We conclude that a proper statistical analysis for rotation curve fitting is crucial for further justification of rotation curve analysis. It is known that the rotation curve analysis 

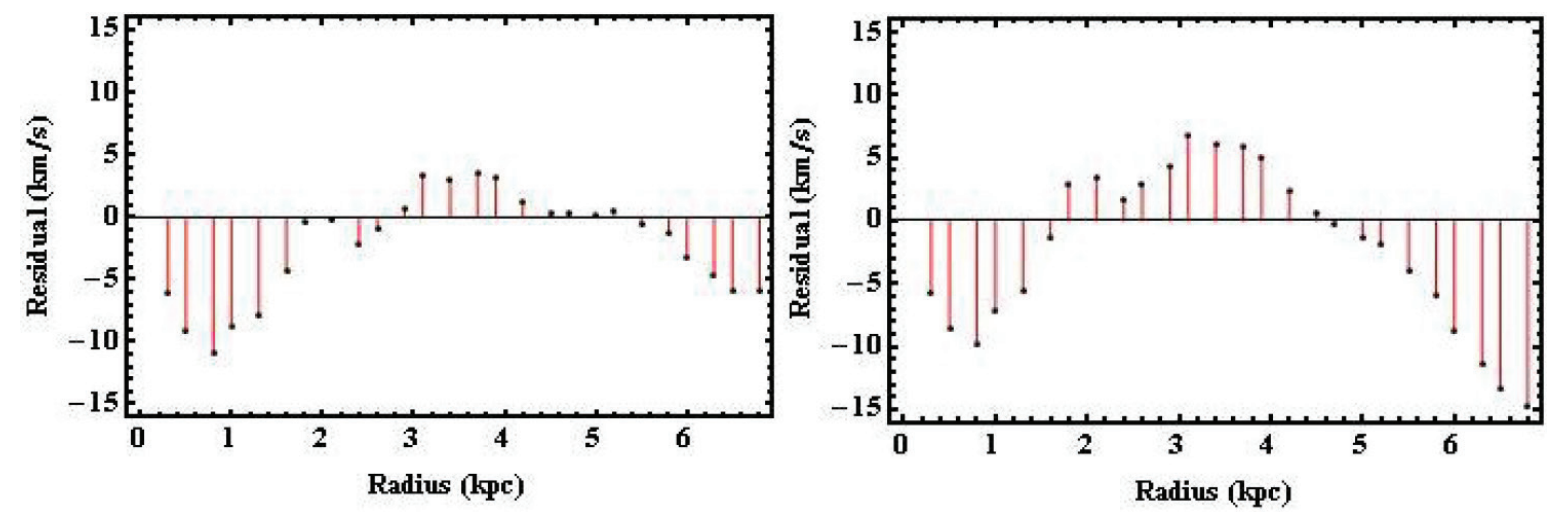

FIGURE 4. (a) The residuals $\left(V_{\text {NewData }}-V_{\text {model } 1}\right)$ plot for rotation curve fit of Burkert profile and

(b) The residuals $\left(V_{\text {NewData }}-V_{\text {model } 2}\right.$ ) plot for rotation curve fit of NFW profile

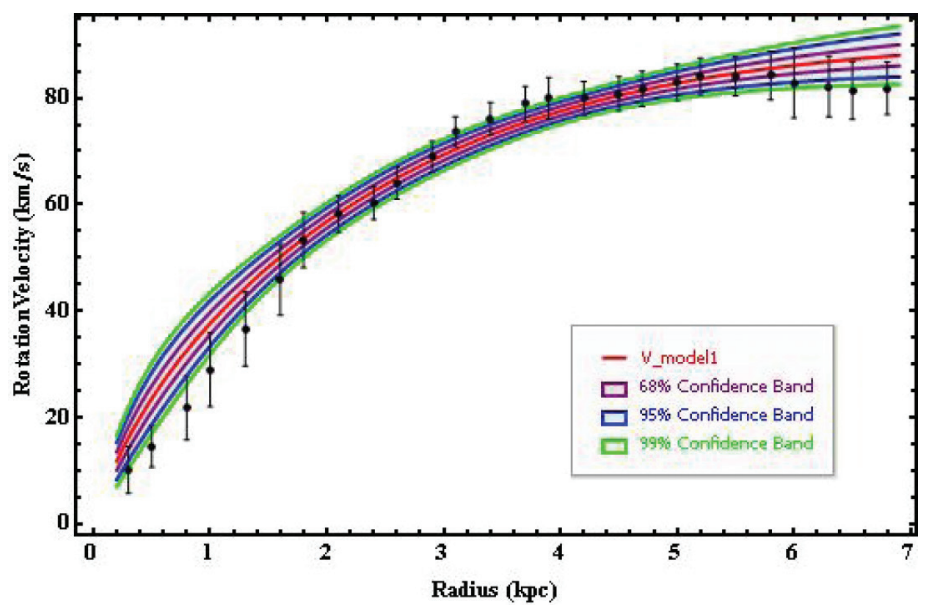

FIGURE 5. The visualization of confidence level band of 68,95 and $99 \%$ with the $V_{\text {model } 1}$ and $V_{\text {NewData }}$

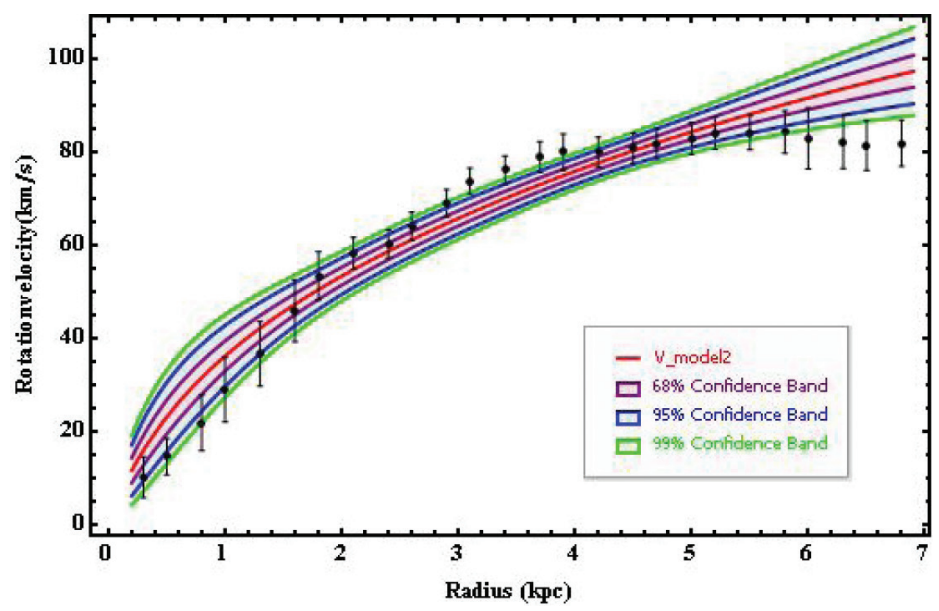

FIGURE 6. The visualization of confidence level band of 68,95 and $99 \%$ with the $V_{\text {model }} 2$ and $V_{\text {NewData }}$

is an important tool to probe the distribution and nature of dark matter in the galaxies. Our approach on gradient method of nonlinear least square fitting shows that the best-fit which is valid out to the last measured point of the observed rotation curve of Orion dwarf galaxy is well in agreement with model function, $V_{\text {model } l}$ compared to $V$ 
This finding can be interpreted as $V_{\text {modell }}$ applied to Burket halo profile is the best model to describe the distribution of dark matter whereby it predicts substantial cored dark matter halo for the Orion dwarf galaxy. It is supported by the value of $\chi^{2}$, plot of $V_{\text {res }}$ and also confidence bands. Hence, we are able to characterize the distribution of dark matter in the Orion dwarf galaxy, which is more towards cored dark matter distribution with halo density, $\rho_{b}=3.9 \times$ $10^{6} M_{\odot} \mathrm{kpc}^{-3}$. Inclusion of the results of the fitting is similar to Frusciante et al. (2012) and leads to the same conclusion even when we consider the different disk scale length. Further, we can apply the problem to the alternative of dark matter called modified newtonian dynamic (MOND) as we did in Hashim et al. (2013), but in different perspectives, that is in statistical perspectives.

\section{ACKNOWLEDGEMENTS}

The authors wish to thank P. Salucci from SISSA, Trieste for bringing this problem and we try to put this into a coherent picture of a statistical point of view. We also acknowledge J.M. Cannon's data of the Orion dwarf galaxy for this study. A personal thank you from N. Hashim to all members of Radio Cosmology Research Group in the Physics Department of Science Faculty, Universiti Malaya, for their continuing support during her study. This study has made use of the Universiti Malaya Research Grant (UMRG) under grant number RG155/11AFR.

\section{REFERENCES}

Bevington, P.R. \& Robinson, D.K. 1969. Data Reduction and Error Analysis for the Physical Sciences. vol. 2. New York: McGraw-Hill.

Binney, J. \& Tremaine, S. 2011. Galactic Dynamics. New Jersey: Princeton University Press.

Burkert, A. 1995. The structure of dark matter halos in dwarf galaxies. The Astrophysical Journal Letters 447(1): L25.

Cannon, J.M., Haynes, K., Most, H., Salzer, J.J., Haugland, K., Scudder, J., Sugden, A. \& Weindling, J. 2010. The stellar and gaseous contents of the Orion dwarf galaxy. The Astronomical Journal 139(6): 2170.

Carignan, C. \& Beaulieu, S. 1989. Optical and HI studies of the 'gas-rich' dwarf irregular galaxy ddo 154. The Astrophysical Journal 347: 760-770.

Côté, S., Carignan, C. \& Freeman, K.C. 2000. The various kinematics of dwarf irregular galaxies in nearby groups and their dark matter distributions. The Astronomical Journal 120(6): 3027.

Freeman, K.C. 1970. On the disks of spiral and so galaxies. The Astrophysical Journal 160: 811.

Frusciante, N., Salucci, P., Vernieri, D., Cannon, J.M. \& Elson, E.C. 2012. The distribution of mass in the Orion dwarf galaxy. Monthly Notices of the Royal Astronomical Society 426(1): 751-757.
Gentile, G., Salucci, P., Klein, U. \& Granato, G.L. 2007. NGC 3741: The dark halo profile from the most extended rotation curve. Monthly Notices of the Royal Astronomical Society 375(1): 199-212.

Hashim, N., Salucci, P. \& Abidin,Z.Z. 2013. The study of rotation curve with Mond for Eso138-G014. Paper presented at the AIP Conference Proceedings.

Longair, M.S. 2008. Galaxy Formation. Berlin: Springer-Verlag.

Navarro, J.F., Frenk, C.S. \& White, S.D.M. 1996. The structure of cold dark matter halos. The Astrophysical Journal 462: 563.

Navarro, J.F., Frenk, C.S. \& White, S.D.M. 1997. A universal density profile from hierarchical clustering. The Astrophysical Journal 490(2): 493-508.

Vaduvescu, O., McCall, M.L., Richer, M.G. \& Fingerhut, R.L. 2005. Infrared properties of star-forming dwarf galaxies. I. dwarf irregular galaxies in the local volume. The Astronomical Journal 130(4): 1593.

Weldrake, D.T.F., De Blok, W.J.G. \& Walter, F. 2003. A highresolution rotation curve of NGC 6822: A test-case for cold dark matter. Monthly Notices of the Royal Astronomical Society 340(1): 12-28.

N. Hashim* \& U.F.S.U. Ibrahim

Centre for Foundation Studies in Science

Universiti Malaya

50603 Kuala Lumpur

Malaysia

Z.Z. Abidin, M.S.R. Hassan \& Z.A. Ibrahim

Radio Cosmology Research Lab

Physics Department, Faculty of Science

Universiti Malaya

50603 Kuala Lumpur

Malaysia

\section{Z.S. Hamidi}

Faculty of Applied Sciences

Universiti Teknologi MARA (UiTM)

40450 Shah Alam, Selangor Darul Ehsan

Malaysia

\section{R. Umar}

Astronomy Unit, Universiti Sultan Zainal Abidin 21300 Kuala Terengganu, Terengganu Darul Iman Malaysia

*Corresponding author; email: norsiahashim@um.edu.my

Received: 15 January 2014

Accepted: 21 August 2014 